\title{
Relationship between body dissatisfaction and social media
}

\author{
Maitrayee Patil ${ }^{1}$, Deepali Bansal ${ }^{2}$, Brijesh Saran ${ }^{3 *}$ \\ 1 Junior Resident, ${ }^{2}$ Senior Resident, ${ }^{3}$ Assistant Professor, Dept. of Psychiatry, Santosh Medical College and Hospital, Ghaziabad, Uttar \\ Pradesh, India
}

*Corresponding Author: Brijesh Saran

Email: sbbxxy@gmail.com

\section{What is body image?}

Body image is the perception that a person has of their physical self and the thoughts and feelings that result from that perception. These feelings can be positive, negative or both. These feeling are mediated by individual factors like ones gender (female), age (13 years -35 years), physical health, and personality. Environmental factors like socioeconomic status, media exposure, type of parenting etc. also play an important role in development of body image.

\section{What is body image dissatisfaction?}

Body image dissatisfaction is the negative inclination towards one's physical body shape where majorly weight is implicated. Body image dissatisfaction towards weight can lead to minor issues like low self-esteem, stress and even extremes of mood disorders like depression, anxiety, and eating disorders.

In this era of technology where internet has turned our existence up and down, it has revolutionised communication to the extent that is now our most and sometimes the only preferred way of communication. But usage of social media constitutes the majority of time spent in internet.

Social media like a coin has two sides. It's pros and cons from being immediate access to information, communicating to your loved ones in any corner of the world to cyber bullying, negative body image, anxiety, depression, unhealthy sleep patterns, general addiction etc.

One of the recent and alarming problem that has come to light is "how social media affects our perception about body image" and its consequences on one's mental health.

According to the latest data, India has the largest number of Facebook users in the world accounting approximately 241 million users which makes the largest user base $(11 \%)$, with maximum number of people belonging to age group of 18-24 years (majorly male).

Facebook, Instagram, Twitter, Snapchat are amongst some of the most widely used social media platforms allowing individuals to create their own personal profiles and share information and photos with users on social their social network. Emerging evidence provides insight into the short and long term effects that social media behaviours may have on its users.

Use of these sites may lead to extreme preoccupation and focus on the physical appearance which in turn leads to poor body image and lowered self-esteem and ultimately body dissatisfaction.
Social media users are frequently exposed to a variety of other profiles, where they can compare their own appearance to friends, relatives and strangers including celebrities. This leads to the user setting unrealistic goals and expectations from one's own profile and pictures.

\section{According to various studies}

An American study which surveyed 227 female college going students, found that young women who spend more time on Facebook/Instagram may feel more concerned and are distressed about their body because they compare their appearance to others.

A study conducted by the Florida House Experience, a health care institution, uncovered that both women and men compare their bodies with those in the media. The same study concluded that $65 \%$ of men compare their bodies to the images they consume on social media and have serious impact on mental health. This is eye opening in view of the common perception that females are ones most affected by body image issues.

There are reports that people who spend time on social networking sites Facebook/Instagram experience more negative and fluctuating mood than those who spend time on other websites used for education, information or news.

\section{Self (ie) Love}

A selfie is a photograph that one has taken of oneself typically taken with a smart phone or webcam and shared via social media.

The most common way that users selectively self present on social media is through the taking and uploading "selfies". Users tend to capture selfies from a flattering angles, using bright lighting, skin- retouching and even photo shopping to make body parts appear flawless, thinner and more attractive.

Taking selfies is a self-oriented action that allows users to establish their individuality and self-importance, it is also associated with personality traits such as narcissism.

Social media present innumerable idealised images of women of being thin, lean/tone, beautiful, photo-shopped women and the "thin ideal" and "muscular" and "athletic ideal" for men are displayed as a normal, desirable and attainable body type for every women or men.

It is in a way conveying the youth to internalize the "thin ideal", "six packs abs" causing them to strive for an unrealistic, unnatural standard of beauty. They feel ashamed when they are unable to achieve it and feel miserable about one self. This has the most dangerous implications in 
vulnerable age group of teenagers and young adults. It may have serious impact on their mental health leading to obsession, mood disorders and even personality disorders.

These predictions are concerning, since high body dissatisfaction is primary risk factor for the development of eating disorders and is correlated with low self -esteem and clinical depression.

Well-known actress and style icon of Bollywood Sonam Kapoor has come forward about the same issue, discusses the facts like how magazines have to retouch her thighs to cover her cellulite. She said "there's lot of effort that goes into making me look his way, it's my job. There are lot of professionals working on me and it takes a lot of money. I don't want girls to think that it's easy or that is how I wake up". She insists, "I want to remind girls that it's okay to celebrate yourself but also to not put so much pressure on yourself".

\section{How to keep social media from damaging your body image?}

1. Be mindful about when and why you are using it, be wise about what you post on your own feeds.

2. Unfollow or unlike pages or feeds that are triggering or cause you to "compare and despair" in terms of food habits or body shape.

3. Follow accounts that encourage healthy, flexible and realistic nutrition and exercise habits.

4. Sleep without phone- as the phone screen emits blue light which leads to dysregulation of melatonin and sleep cycle.

5. Use a table clock- Avoid compulsive checking of mobile phone first thing in morning, which further takes one on a social media spree.

6. Decrease screen time and the amount of time spent on social media by involving yourself in more productive activities or pursuing hobbies.

7. Have Digital detox periods, which includes scheduled regular multi-day breaks from social media.

Note: Here the few apps which could help you to curb social media use and decrease your app/screen usage time.

1. In moment

2. Space

3. App detox

4. Off the grid

5. Anti social 